\title{
Ab Initio Nonadiabatic Surface-Hopping Trajectory Simulations of Photocatalytic Water Oxidation and Hydrogen Evolution with the Heptazine Chromophore
}

Xiang Huang and Wolfgang Domcke*

Department of Chemistry, Technical University of Munich, D-85747 Garching, Germany

\section{Supporting Information}

1. Hz atom numbering

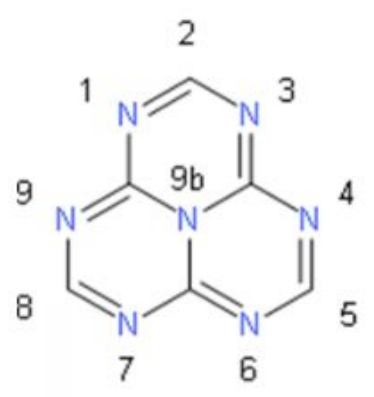

Figure S1. Atom numbering of $\mathrm{Hz}$ molecule ${ }^{1}$. 


\section{Supporting figures}

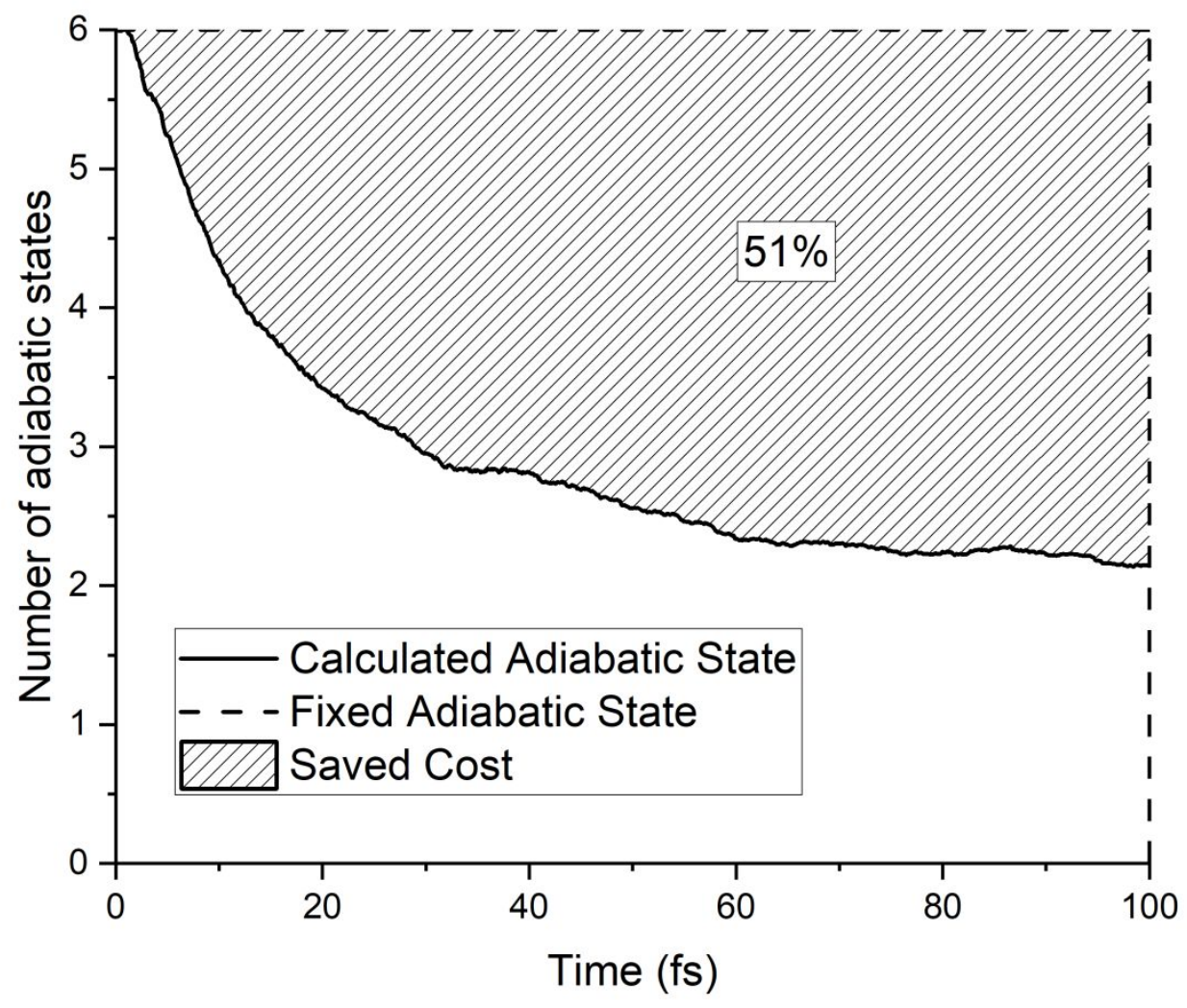

Figure S2. Time evolution of the number of adiabatic electronic states included in the calculation for the $\mathrm{Hz} \cdots \mathrm{H}_{2} \mathrm{O}$ complex. The computational cost is roughly proportional to the number of adiabatic states. The shaded area approximately represents the saved computational cost compared with a calculation with a fixed number of adiabatic states $(\mathrm{N}=6)$. 

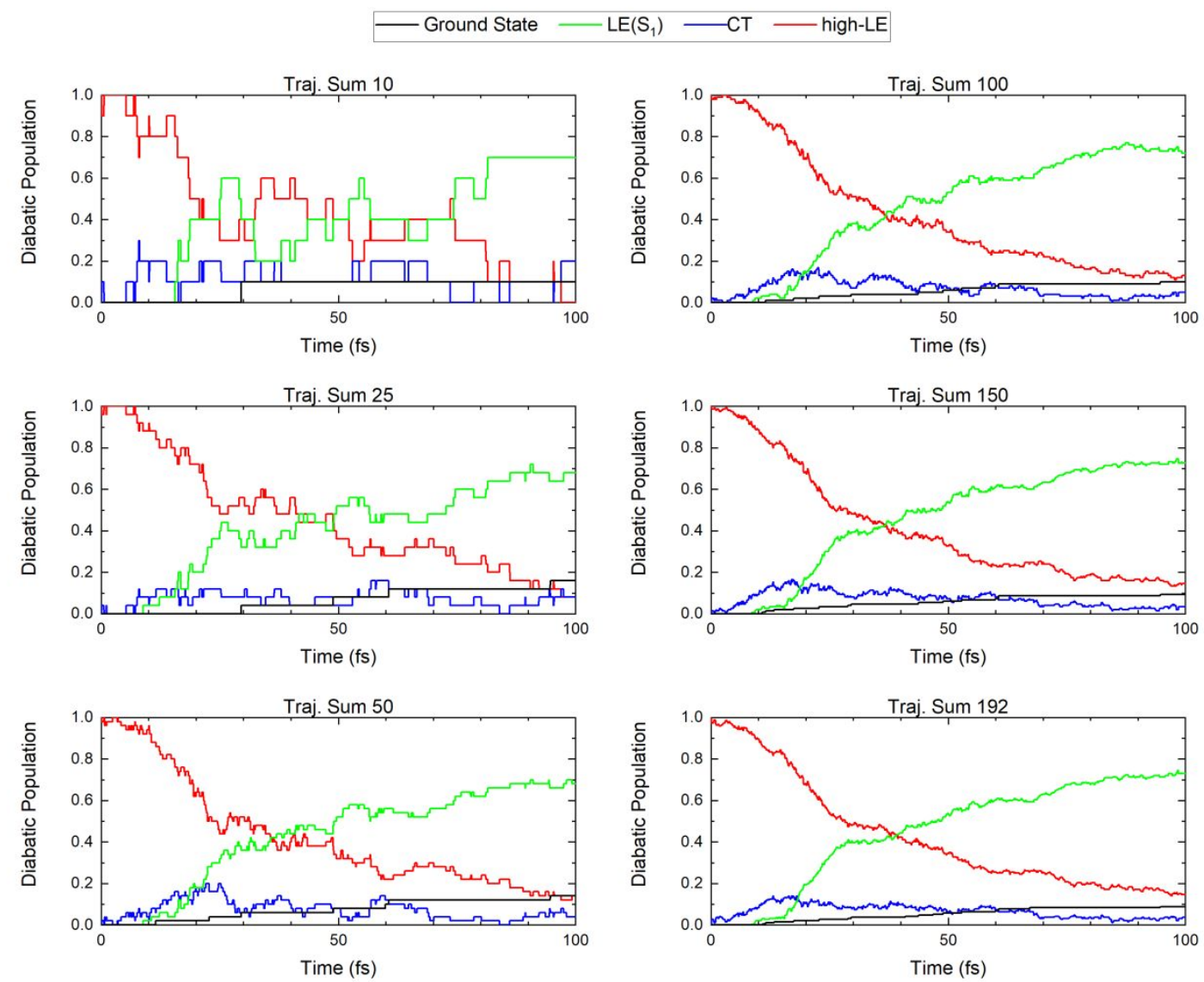

Figure S3. Time evolution of the quasi-diabatic electronic populations of the $\mathrm{Hz}^{\cdots} \mathrm{H}_{2} \mathrm{O}$ complex with different sizes of the trajectory ensemble up to $100 \mathrm{fs}$. The $\mathrm{S}_{0}$ population is colored in black, the $\mathrm{S}_{1}$ population in green, the CT population in blue and the population of the high-lying LE states in red. 


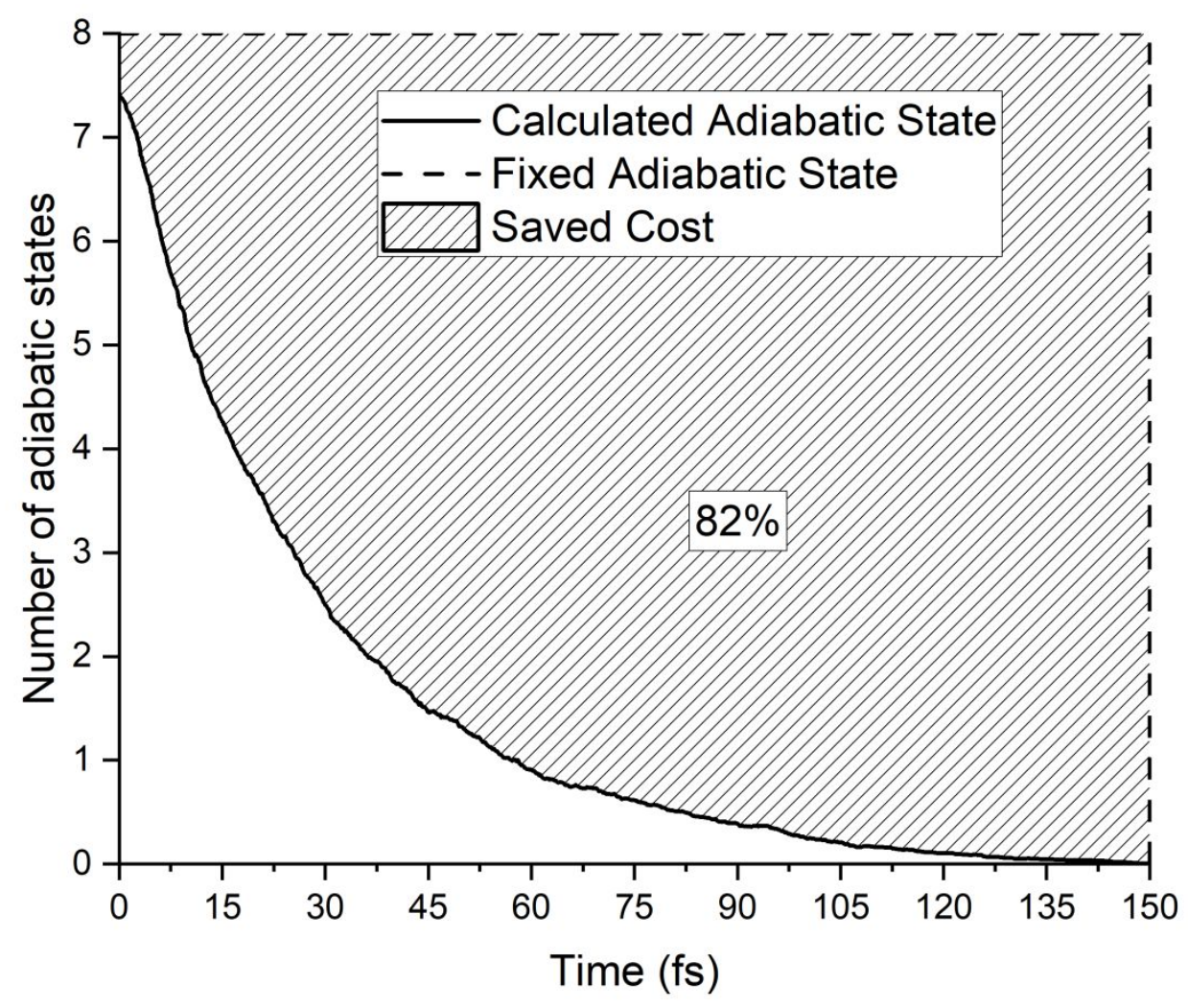

Figure S4. Time evolution of the number of adiabatic electronic states included in the calculation for $\mathrm{HzH}_{2}$. The computational cost is roughly proportional to the number of adiabatic states. The shaded area approximately represents the saved computational cost compared with a calculation with a fixed number of adiabatic states $(\mathrm{N}=8)$. 


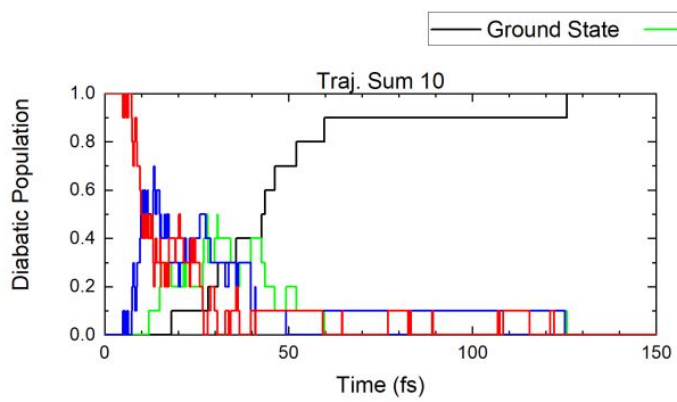

low- $\pi \pi^{\star} \longrightarrow \mathrm{CT} \longrightarrow$ high $-\pi \pi^{\star} / \mathrm{n} \pi^{\star}$
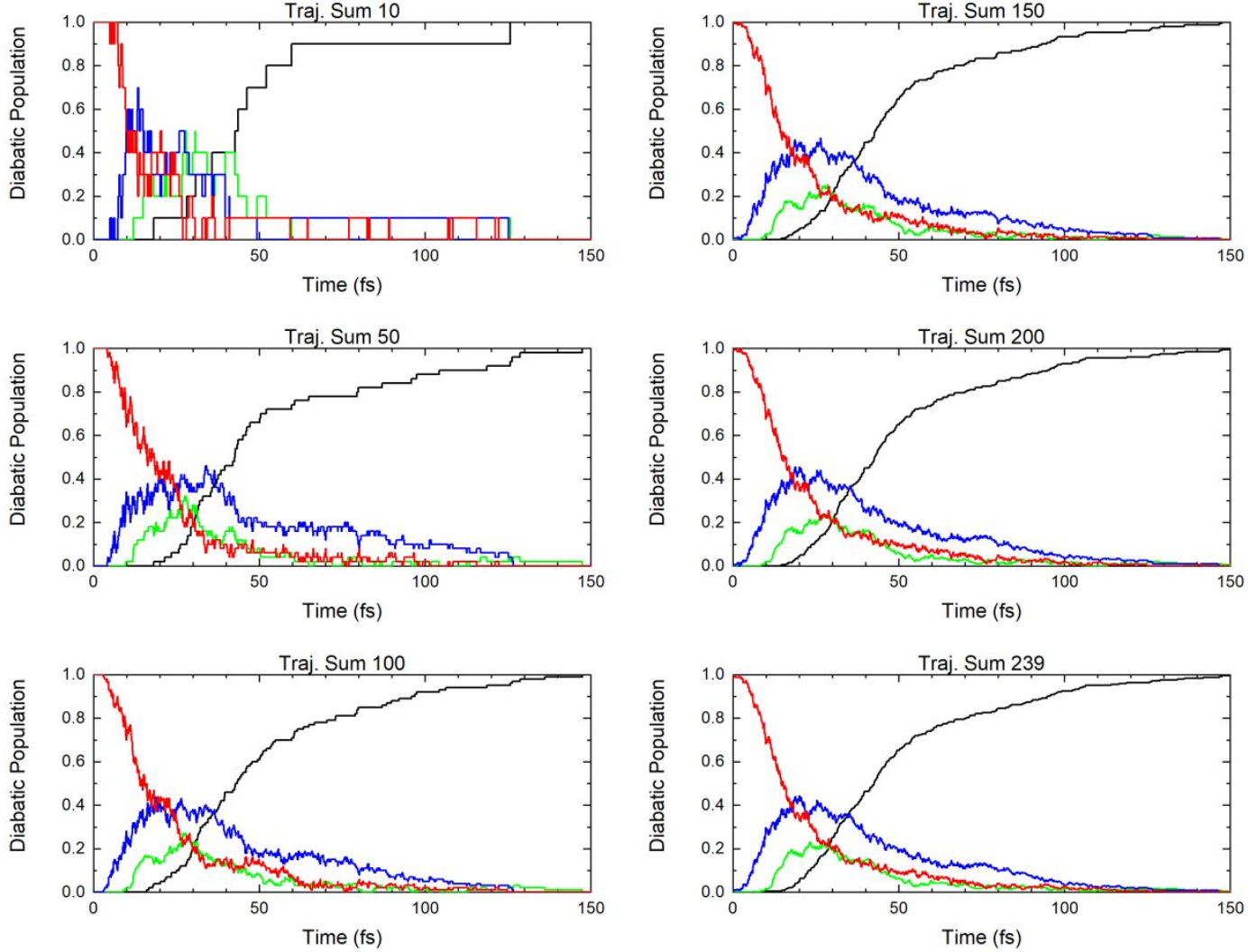

Figure S5. Time evolution of the quasi-diabatic electronic populations of $\mathrm{HzH}_{2}$ with different sizes of the trajectory ensemble up to $150 \mathrm{fs}$. The $\mathrm{S}_{0}$ population is colored in black, the population of the lowlying $\pi \pi^{*}$ states in green, the population of the $\pi \sigma^{*}$ states in blue and the population of the high-lying $\pi \pi^{*} / \mathrm{n} \pi^{*}$ states in red. 


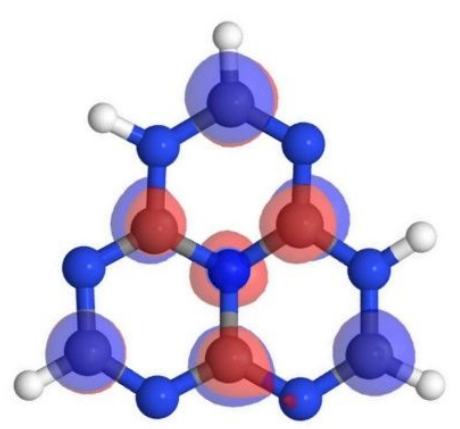

(I)

$+0.08 \mathrm{eV}$

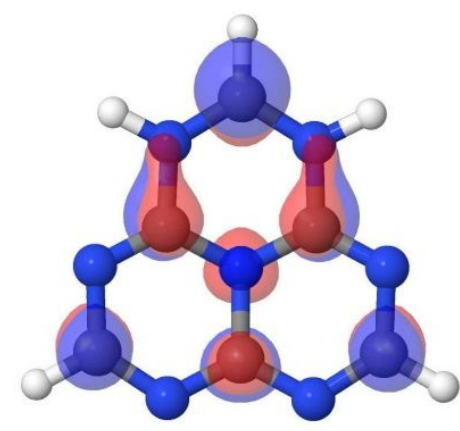

(III)

$+0.37 \mathrm{eV}$

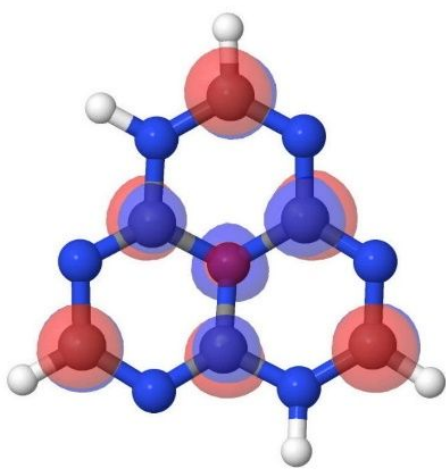

(II)

$+0.00 \mathrm{eV}$

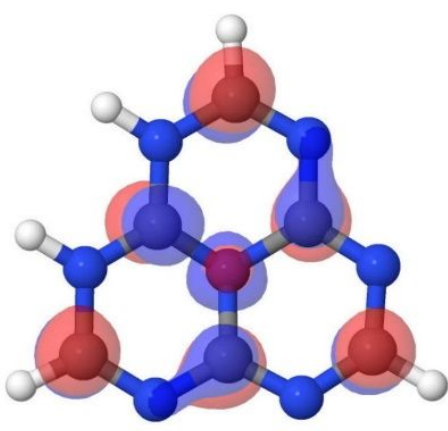

(IV)

$+0.89 \mathrm{eV}$

Figure S4. HOMOs of the tautomers of $\mathrm{HzH}_{2}$.

\section{References}

1. Hosmane, R. S.; Rossman, M. A.; Leonard, N. J., Synthesis and Structure of Tri-S-Triazine. J. Am. Chem. Soc. 1982, 104 (20), 5497-5499. 